\title{
Faire chanter la parole, faire parler la musique. Généalogie d'une instauration réciproque
}

\author{
Antoine HENNION ${ }^{1}$
}

\section{Summary}

There is no evidence in taking for granted the Great Divide between musica and parola: Emerging genres and practices constantly reinvent ways of redefining and connecting those entities. By presenting contrasted ways of singing words, from ethnic ritual performances to religious cult, from opera to French song or rap, this paper outlines ways in which all along history, speaking and singing have re-formed each other through contrasted relationships. Following Foucault's plea for "genealogy" and Certeau's concept of the "inversion of the thinkable", it thus aims to address music history without writing it backwards by drawing on categories that themselves are products of this history. To sketch such a genealogy of its basic elements may help us rebuild our ways of making music of the past present again.

Par rapport au thème du colloque Lingue parlate, lingue cantate (2019) dans le cadre duquel les idées suivantes ont été discutées, cette contribution revient sur les multiples façons dont, depuis les temps et dans les lieux les plus lointains, des paroles ont été chantées. Mais il ne s'agit pas des différentes manières de "déposer de la musique le long d'un texte ", comme dirait Victor Hugo. Une telle formulation tient déjà trop pour acquis les termes de ce couple sensuel parole et musique, dont il ne s'agirait que de suivre le pas de deux tout au long de l'histoire : ce serait les prendre d'emblée pour ce qu'elles sont devenues. La voix parlée et la voix chantée, pour utiliser ces termes à la fois plus proches et plus flous, n'ont cessé de se former l'une l'autre, et de changer ainsi de nature, l'une grâce à l'autre, l'une contre l'autre. Dans le fil du concept de généalogie (cf. Foucault 1994), mon propos est à l'inverse de saisir la voix, les mots, les notes, la musique enfin, comme des catégories performatives. Ce ne sont pas des catégories analytiques dont nos savoirs modernes pourraient décliner les rapports historiques : elles ont fait se réaliser ce qu'elles nomment. ${ }^{2}$ 


\section{Une conception historique de la sensibilité, une conception sensible de l'histoire}

Ainsi, en m’appuyant sur les liens qui se sont forgés entre parole et musique, mon objectif est double, sans qu'il soit si facile de déterminer lequel sert de moyen à l'autre : dans un sens, essayer de rendre l'histoire sensible, de la faire passer par nos oreilles, et pas seulement par nos cerveaux ; dans l'autre, de rendre notre écoute véritablement historique. L'art musical semble se prêter particulièrement bien à l'exercice, lui qui doit sans cesse rejouer les œuvres du passé, et non seulement les accrocher au mur ou les poser dans un musée. Mais justement, est-il possible non pas d'écouter ces œuvres ‘ comme nous l'entendons `, mais au contraire de prêter l'oreille à ce qu'elles ont pour nous de radicalement étranger ? Non pas les faire nôtres, les apprivoiser, mais mieux sentir ce que nous avons définitivement perdu d'elles, avec la disparition de leurs conditions de production ? Et peut-être de là, en réinventer une autre écoute?

Comment donc écrire à rebours sur des musiques passées, c’est-à-dire sans projeter sur elles des notions que cette histoire même a forgées? Le problème historiographique que cela soulève est l'un des objets de cet article. Mais d'abord, un petit préalable, pour éviter toute équivoque à propos du mot ‘ performatif .. En glissant ainsi des actes de langage aux réalités elles-mêmes, il a changé de sens : pour le dire avec de grands mots, il est passé d'une acception linguistique et institutionnelle à une conception ontologique et topologique. L'intérêt de la définition initiale proposée à propos des actes de langage et du rapport des mots aux choses par la pragmatique de l'énonciation (cf. Austin 1970) résidait dans la force de l'opposition binaire entre des énoncés performatifs, qui ‘ font ce qu'ils disent >, et la plupart des autres énoncés, seulement constatifs. ${ }^{3}$ La conception de la performativité évoquée plus haut est au contraire continuiste et ontologique : elle défend qu'à un degré plus ou moins fort, ce sont tous les êtres qui se font et se font faire. Cette vision ouverte d'un monde ‘ toujours en train de se faire , fait de couches de réalités hétérogènes mais connectées, renvoie plutôt au pragmatisme radicalement empiriste et pluraliste de William James (James 1909, 226 ; Hennion 2015). ${ }^{4}$ Elle implique aussi qu'il n'y a pas de point de vue extérieur ou atemporel d'où observer et analyser les réalités : on ne peut les saisir dans l'absolu mais seulement en les < expériençant `: en les éprouvant et en les mettant à l'épreuve. Elles se font elles-mêmes, appuyées sur toutes les autres : quoi de plus musical ! Étienne Souriau avec son idée de l'، œuvre à faire , précisément inspirée du cas des œuvres d'art mais nullement réservée à elles, prolongeait cette vision en lui donnant une portée éthique et esthétique d'une rare ampleur : chaque être dépend de la sollicitude de tous les autres (cf. Souriau 1956 ; Hennion/Monnin 2015).

Une telle conception peut sembler abstraite, en dépit de sa prétention à s'inscrire dans le concret de chaque moment. Mais ici, le cas de la musique est d'un intérêt tout particulier, à cause du rapport à la fois sensible et historique de tout musicien au passé. L'idée qu'une œuvre soit ‘ toujours à faire ’, qu’il n’y ait pas de passé sans qu'il soit actualisé dans le présent, c'est l'évidence pour lui, qu'il joue ou qu'il écoute. Et réciproquement : ces objets d'un passé sans cesse redonné comme présent n'existent que s'ils sont à nouveau ressentis, même si c'est sur un tout autre mode que par le passé. 


\section{De la musique avant la musique?}

Comment, sur ces prémisses, parler de la musique d'avant que le mot musique ait un sens ? Comment évoquer la parole alors que son mélisme se distingue encore à peine du cri, de l'appel, de la plainte ou du grognement expressif dont elle s'extrait ? Certes, nous n'avons pas d'enregistrement des échanges vocaux entre Néanderthaliens, mais il n'est pas nécessaire de remonter si loin. Dans le temps comme dans l'espace, tant l'histoire que l'ethnologie nous permettent d'avoir accès, même très indirectement, à d'autres états des choses que les nôtres : pour le dire de façon pompeuse, d'entrevoir des temps, des lieux et des pratiques d'avant le grand partage entre parole et musique (cf. Rouget 1985 ; Despringre, 2019). Aujourd'hui même, les contrastes demeurent, entre des genres qui jouent sur la proximité ou sur la distance entre parole et musique : si la chanson se situe juste à leur frontière, comme pour conserver précieusement cette fragilité de l'entre-deux, le lied allemand, l'opéra italien ou la mélodie française ont au contraire sur-élaboré chacun de ces deux éléments pour ensuite mieux les combiner, ce qui est très différent - qui confondrait leurs constructions savantes avec une chanson populaire?

Le défi n'est pas simple. Tout notre vocabulaire est là pour nous faire penser de façon distincte des choses qui n'étaient pas clairement différenciées dans les réalités et pour les acteurs auxquels nous nous référons : c'est-à-dire des performances orales, rythmées, chantées, scandées, dans lesquelles la fonction d'une manifestation collective (magique, rituelle, sacrée, religieuse, politique, festive, amoureuse, poétique...) primait sur l'identification technique de composants spécifiques, tels que la danse, la parole et la musique. Nous-mêmes associons très facilement les trois aujourd'hui. Mais, comme je le signalais, ce n'est pas parce que nous les confondons : à l'inverse, c'est qu'une longue histoire s'est chargée de les dissocier pour nous permettre de les recombiner.

Je vais donc reprendre cette histoire en musique, sans prétendre pouvoir faire plus ici que suggérer l'intérêt de l'aborder sur le mode généalogique. Dans une première partie, plus théorique mais nécessaire, avant de l'esquisser sur une série de cas contrastés, je vais discuter de l'intérêt et de la possibilité même d'une telle façon d'écrire l'histoire. Car comment faire, pour ne pas prendre l'histoire de la musique pour le simple antécédent de son état actuel, ce qui revient à écrire l'histoire en marche arrière ? Dans les pas du Foucault de l'Archéologie du savoir (1969), Michel de Certeau l'a dit mieux que personne. C'est ce qu'il appelait l'« inversion du pensable ", dans son formidable livre sur l'écriture de l'histoire (Certeau 1975, 131, à propos de la religion au XVII e siècle) : nous croyons lire le passé avec nos catégories modernes, alors que c'est le passé qui les a produites pour nous. Cela veut dire qu'il n'en disposait pas et ne peut être compris à travers elles, mais aussi qu'elles viennent de lui : autrement dit, c'est lui qui nous écrit.

Écrire l'histoire, non seulement ce n'est donc pas nous transporter dans le passé, cela veut presque dire l'inverse : mieux réaliser cette impossibilité, prendre conscience de notre irrémédiable distance à lui (cf. aussi Veyne 1971, ou, en référence à Foucault, Boquet/Dufal/ Labey 2003). En faire un passé en effet, mais en un sens très actif, de le transformer en notre 
passé, et à la différence du souci de respecter la tradition, par exemple, qui, elle, ne cesse de changer sans changer, sans prendre acte de ce qu'elle n'est jamais la même (cf. Lenclud 1987)..$^{5}$ L'historien n'écrit que sur une absence, le passé, cet « absent de l'histoire " (Certeau 1973), non pour simuler sa présence mais au contraire pour l'installer aujourd'hui dans ce qui par ce geste même en fait notre passé. Ce « notre ` signale bien le caractère actuel dudit passé, qui est donc par là-même ouvert, pluriel, toujours à refaire. C'est pourquoi les historiens recommencent indéfiniment à l'écrire, l'histoire n'est jamais établie : si les historiens se doivent de nous présenter le passé, et de sans cesse le représenter, c'est justement parce qu'il nous est devenu définitivement étranger.

\section{La musique, l'art de faire du passé une présence}

Il s'agit donc bien toujours de rendre le passé présent, mais les mots sont trompeurs : l'expression n'est pas à comprendre au sens où le passé dirait « je suis là ! " comme on répond "présent! » à l'école, mais au sens de conférer au passé une autre présence, ou plutôt une présence autre, qui lui conserve sa distance, sa radicale étrangeté. Ne pas faire comme si nous vivions au passé, bien sûr, mais ne pas non plus croire le contempler tel qu'il était alors, comme si passé et présent étaient des réalités fixes, de simples points distants mais mesurables dans un espace-temps global, homogène et continu qui nous contiendrait tous. C'est seulement depuis notre temps que nous pouvons et devons prendre la double mesure de l'altérité du passé et de sa perte - non pour l'oublier, mais parce que c'est la condition pour rendre vivante aujourd'hui une autre expérience du passé, toujours renouvelée, à travers ce que nous avons hérité de lui. Encore un sens du mot présent : oui, vu ainsi, le présent est bien un don, le cadeau que les siècles passés nous chargent de reprendre.

Dite ainsi, la chose semble un peu ardue, ou du moins il n'est pas si facile d'en voir les implications concrètes. Pourtant, bien loin d'être abstraites, ces idées sont au contraire très sensibles, et comme je l'ai signalé, la musique, toujours à rejouer, est l'objet idéal pour faire sentir l'abîme qui sépare, qui oppose même terme à terme ces deux versions du rapport entre présent et passé. Plus que les tableaux qui, ‘ eux-mêmes ', semblent appartenir à leur temps et, par leur fixité même illusoire, renvoyer la recherche sur les diverses façons dont nous les voyons à une sociologie de la réception, l'exemple de la musique aide à comprendre ce que signifie à la fois notre distance au passé, et la façon dont nous pouvons en faire un présent.

Un simple concert suffit en effet à éclairer ce qui peut sembler obscur dans cette formulation : Beethoven a beau avoir écrit il y a deux siècles, le pianiste avoir travaillé mille fois sa sonate, et nos oreilles en être familières, les doigts de l'interprète qui tremblent avant d'entrer sur scène lui rappellent qu'il n'y a que le présent. Seul compte un rendre présent. Beethoven et les autres n'existent que parce qu'on en refait continûment l'expérience. Même et surtout appuyée sur tout ce qui nous fait, l'expérience n'existe jamais qu'au présent (cf. Hennion 2012). Mais il faut aussitôt ajouter que ce présent n'est pas un point sur une ligne : tissé de multiples couches de réalité entrecroisées, il est pluriel, ouvert, indéterminé. 
Pour la musique, ce trait ne tient pas seulement au caractère vivant, comme on dit assez justement, de toute performance, il vaut tout autant pour l'écoute d'un enregistrement ou la lecture d'une partition. Quelle que soit sa forme, l'expérience de l'écoute est toujours à refaire. Il s'agit bien, plus globalement, de ce qui a peu à peu constitué collectivement, et avec ses contrastes, notre écoute actuelle. Prenons l'exemple de Bach. Bach n’a pas écrit le BWV, ce catalogue n'appartient qu'à nous! Qu'y a-t-il de commun entre, d'un côté, un bourgeois de Leipzig allant prier à l'office le dimanche, qui entend la parole de Dieu mise en valeur par une cantate que Bach a écrite dans ce but, en reprenant s'il le faut du matériel venant d'autres ouvres, et que l'un et l'autre ne pensent pas même réentendre... et, de l'autre, notre propre écoute, celle du père de la musique, dont nous connaissons vingt versions d'œuvres cataloguées et apprises dès l'enfance, que tous les musiciens entre lui et nous ont repris dans leurs œuvres et intégré dans nos oreilles, et qu'une chaîne industrielle et commerciale ininterrompue enregistre, vend et diffuse en continu à des amateurs désormais plus préoccupés de leur plaisir musical que du contenu religieux de ces paroles (cf. Fauquet/ Hennion 2000 ; Hennion 2010) ?

Comment mieux comprendre que c'est précisément toute cette machinerie mise en place pour rendre présent le passé qui nous en éloigne en même temps le plus radicalement ? Et aussi, loin d'une nostalgie hors de propos, que ce sont ces couches de passés continûment refaits présents qui font la richesse de notre présent... Oublions donc notre définition actuelle de la musique, ce serait écrire l'histoire à l'envers. Nous n'aurons jamais les oreilles d'un paysan, d'un moine ou d'une gente dame du Moyen Âge. Nos façons d'entendre et de penser la musique n'étaient pas les leurs. Or, pour répondre au défi proposé, celui de tenter une approche généalogique de la musique, l'exemple du rapport parole-musique est particulièrement éclairant : il aide à suivre à la trace cette chose étrange qu'on appelle musique en train de se dégager lentement de ce dont elle est née, le rite social, les accents des voix, le mouvement des corps, sans faire l'anachronisme de lire ces états intermédiaires à partir de leur état futur. Ou comment filer l'engendrement et l'augmentation réciproques de la musique et de la parole, chacune ajoutant à la puissance de l'autre...

Comme je l'indiquais, un tel programme, trop imposant pour être développé dans ce cadre limité, ne sera ici qu'esquissé, sur un mode plus expérimental et hésitant que bien assuré.

\section{Vous avez dit Africains ?...}

Commençons par deux musiques contrastées. Deux musiques africaines, au sens où leurs interprètes le sont... Et pourtant! La première est un enregistrement réalisé par Vincent Dehoux, un disciple de Simha Arom, l'ethnomusicologue spécialiste des polyphonies et des polyrythmies de Centre-Afrique. Le second, le début d'Opéra Puccino, est le premier enregistrement en solo d'Oxmo Puccino, alias Abdoulaye Diarra, né à Ségou au Mali en 1974 et devenu depuis l'un des piliers du rap français. 
Écoute 1

https://archive.org/details/8josquinmilleregretz/1-Intro+Banda+Gbambiya+Nginza+ngiriki. mp3

Banda Gbambiya (République centrafricaine) : "Nginza ngiriki », enregistré par Vincent Dehoux.

Il s'agit de chants de village en Centre-Afrique, enregistrés selon la méthode sophistiquée de Arom (1985), par re-recording des musiciens. Le cas des musiques africaines, partie intégrante d'inlassables rituels - le village qui se rassemble, la danse, les herbes, la transe, les femmes qui chantent... -, est d'un abord délicat, par rapport à nos catégories : alors même que le mot musique n'existe pas dans ces sociétés, les instrumentistes n'en sont pas moins sinon des professionnels, en tout cas des spécialistes ayant reçu une vraie formation, notamment à cause de la complexité rythmique de ces musiques, à travers une longue complicité avec des anciens, dans une relation mystique de type maître-disciple. Quant aux performances, elles sont indissociables de la vie collective : fête, enterrement, mariage, maladie, vente de troupeaux, sécheresse, il y a musique si et seulement si le village réuni célèbre, pleure ou combat quelque événement. Arom parlait d' "une musique populaire, orale, anonyme, sans date, collective et à vocation interne " (vol. 1, 56, ital. orig.). Dans ces conditions, trancher pour savoir si l'on a affaire à du social, à de la musique, à une performance, à un rite, etc., n'a pas grand sens. En même temps, tous les ingrédients de la musique sont bien présents, et ils le sont à un niveau très élaboré : des rythmes, des harmonies, des voix, des instruments, plus des textes rituels, chantés par les femmes, qui elles ne sont pas professionnelles - il y a donc même des ‘ amateurs `.

Ces éléments musicaux, on peut en faire l'analyse : c'est la démarche qu'a suivie Arom, en montrant comment les voix se syncopent les unes sur les autres, etc., notamment pour comprendre comment, sans écrit ni code explicite, des polyphonies peuvent néanmoins suivre des règles, et pour prouver que l'analyste extérieur, lui, peut les expliciter. En l'assumant pleinement, Arom analyse ces musiques avec ses propres catégories, et ne se soucie pas de savoir si c'est bien de musique qu'il s'agit, ou plutôt d'explorer le sens de ce mot c'est-à-dire la question même que le présent article soulève. Les ethnologues lui ont souvent répliqué en lui renvoyant la dimension sociale de la musique : les membres de la tribu vivent l'expérience ensemble, ils la lient à leurs ancêtres, aux rituels et à leurs fonctions, elle les définit et les fait exister. En négligeant ces aspects, ne risquait-il pas de rater l'essentiel ? Mais ce n'est pas la piste que je vais suivre. Je partage leur interrogation, non sa formulation, qui nous enferme dans une querelle de double : choisissez votre camp, ou bien la musique est une réalité autonome, ou bien elle n'est qu'une construction sociale... Et si au lieu d'être des réponses, ces deux mots, la musique et le social, étaient plutôt eux-mêmes des partages ' toujours à faire ', indéterminés, se formant l'un l'autre? 


\section{Écoute 2}

https://archive.org/details/8josquinmilleregretz/2-OxmoPuccinoIntro.mp3

Oxmo Puccino (alias Abdoulaye Diarra) : Intro ("Visions de vie ») d'Opéra Puccino, rap de 1998.

Sans être un connaisseur de cette musique, je mets délibérément en regard de ces chants rituels la musique d'un rappeur franco-malien, Oxmo Puccino, que ce titre, Opéra Puccino (avec la plaisanterie Puccino/Puccini), a fait connaître. Le rap constitue un cas très intéressant à propos du rapport parole-musique. Texte plus épelé ou débité que chanté, sons et rythmes très modernes, américains, électroniques, on a là deux fois une sorte de retour arrière, tout à fait assumé : d'abord sur le plan musical, vers cette parole psalmodiée fuyant la mélodie, donc cherchant à retrouver un entre-deux entre parole et chant ; et puis par rapport au français, avec ce retour vers « notre > langue, et non l'anglais qui a dominé la pop et le rock, y compris francophones - ce < notre > étant lui-même ambigu, équivoque : estce le français du Mali, celui des parisiens, celui des banlieusards ? Par opposition au rock, il y a l'idée que la parole et la musique chantée, ce n'est pas si différent, dans la recherche d'une sorte de scansion, d'un rythme, et par là d'une africanité reconstituée et revendicative. En même temps, par rapport au village africain, tout cela est réalisé, produit plutôt au sens anglais du mot, dans un dispositif collectif complètement opposé, puisque même si Oxmo Puccino est très engagé, il s'agit d'une vedette du show-business, enregistrée, mise sur internet, vendue par les maisons de disque, qui circule sur des réseaux commerciaux, politiques, identitaires. D’une certaine façon, il incarne le comble de la modernité occidentale. Reste que le rap a aussi réussi à retisser le lien entre une expression corporelle, musicale, verbale, animée, et une parole politique identitaire, fortement revendicatrice et provocante, exprimant des problèmes sociaux et raciaux : les banlieues françaises ont su se réapproprier ce qu’ont inventé les banlieues américaines, un peu comme si tous ces aspects trop distincts s'étaient déchirés à force de s'autonomiser, et qu'il fallait les raccommoder, retrouver leur force en leur redonnant une unité expressive.

Entre ces deux extraits, donc, voici un premier contraste : d'un côté, sur le versant africain, un monde où la musique est omniprésente, sans être identifiée comme telle; de l'autre, sur le versant occidental, un monde où elle est omniprésente aussi, mais où au contraire on aurait du mal à la percevoir sans considérer que c'est de la musique qu’on écoute. Autre contraste : d'un côté un ethnomusicologue qui, défenseur acharné d'un positivisme méthodique et analytique, laisse aux ethnologues les aspects rituels, sociaux et religieux des pratiques musicales pour revendiquer la nécessité d'une analyse spécifique, purement technique, de la musique ainsi produite, par des peuples qui ne faisaient pas ces distinctions ; de l'autre, un musicien franco-malien qui part de l'état inverse des choses, d'une société où règne la séparation bien ordonnée de nos réalités modernes, pour jouer à fond de leur efficacité, et ranimer ce qu'en réalité tout nouveau mouvement artistique ou culturel actualise : le lien particulier, toujours à refaire, entre un mode d'expression et une réalité inédite qui cherche à s'exprimer. 
Que peut donc exprimer la musique ? Ces croisements inversés entre l'homme moderne et l'Africain, en un sens aussi improbables l'un que l'autre, en outre imprégnés par la place très particulière qu'occupe l'Afrique dans l'imaginaire européen - le primitif, ou l'origine? le sauvage, ou le vif ?... - me semblent bien indiquer le chemin à suivre, si l'on veut reprendre plus précisément la question que je posais : celle des relations de formation réciproque de la parole et de la musique, ou pour le dire plus justement, de la pulsation irrégulière entre des moments où, dans la recherche d'une expression collective, elles luttent pour se distinguer, et des moments où elles s'appuient l'une sur l'autre pour se renforcer.

\section{...mélodies sans âge, médias modernes...}

Écoute 3

https://archive.org/details/8josquinmilleregretz/3-KeyrouzExapostilarion.mp3

Sœur Marie Keyrouz / Marcel Pérès : « Exapostilation de l'Office de Mardi Saint », liturgie de l'église grecque, vers l'an 600 .

Nous revoici revenus en arrière, d'abord vers l'an 600 dans une église grecque, au nord de la Méditerranée. Si disparaît l'effet ésotérique des Pygmées (ou des rappeurs...), reste l'évidence d'une sorte d'état premier de la musique, avec ce qu'on appelle le grégorien (un peu vite : cela dure des III-IV ${ }^{\mathrm{e}}$ siècles au XII ${ }^{\mathrm{e}}$ ). Justement, Marcel Pérès et Sœur Keyrouz ont repris ces chants des tout premiers siècles de notre ère dans les églises, grecques, syriaques, arabes, ici byzantines du $V^{\mathrm{e}}$ siècle. Se rassembler en présence de Dieu, une mélopée qui récite des textes bibliques : on voit combien le mot musique est inutile, ou trop fort, il s'agit d'une prière ; et inversement, on ne ‘ dit > pas une prière sur le ton d'une conversation. Voici une de ces formes où, plus que de se mêler, paroles et musique forment une seule et même chose. $^{6}$

\section{Écoute 4}

https://archive.org/details/8josquinmilleregretz/4-OumKalsoum-AfdihiIntro.mp3

Oum Kalthoum : «Afdihi in hafidza el hawa », chanson arabe, 1928.

Oum Kalthoum, juste après Sœur Keyrouz : une chanteuse égyptienne du XXe siècle, ou plutôt $L A$ chanteuse du monde arabe, de la pointe du Maroc aux confins de l'Iraq et au-delà, de l'Égypte à Barbès, à Paris. Je l'ai placée là pour secouer notre vision à la fois linéaire et ethnocentrée du temps. Cette variabilité même, cette conscience d'un temps qui progresse et laisse son passé derrière lui est la nôtre. Ailleurs priment la circularité infinie du temps et la continuité d'une identité collective, même imaginaire. Ces façons incantatoires de moduler le chant semblent ainsi traverser les siècles, alors même que leurs supports matériels et les sociétés qui les produisent ne cessent de changer : le déroulement de l'histoire n'est pas le même pour tous. Oum Kalthoum est d'autant plus intéressante qu'à côté de ces mélodies 
arabisantes sans âge, avec le même talent et le même succès elle a aussi fait ce qu'on peut qualifier de pure musique de variété, telle une Dalida arabe (pour ses fans) : les fils du présent sont eux aussi entremêlés.

\title{
Polyphonie et écriture
}

\author{
Écoute 5 \\ https://archive.org/details/8josquinmilleregretz/5-SanctusCorseXVIIIe.mp3 \\ Ensemble Organum / Marcel Pérès : "Sanctus ", chant grégorien retranscrit par les Francis- \\ cains en Corse au XVIII .
}

Ce «Sanctus » corse du XII e siècle relève donc, cette fois, du grégorien tardif. Mais surtout, la version qu'a reconstituée l'ensemble Organum est celle qui avait été revue par les Franciscains en Corse. Marcel Pérès a eu la bonne idée de revenir à leurs manuscrits du XVIII ${ }^{e}$, alors qu'ils veulent à la fois faire ce que leur demande leur ordre monastique, c'est-à-dire inculquer le catholicisme orthodoxe à ce peuple qui l'est bien peu, mais aussi le combiner aux façons traditionnelles très particulières de chanter des Corses, qu'ils recueillent au prix de tout un travail d'ethnomusicologie avant la lettre. L'effet produit est très loin du grégorien de Solesmes, c'est-à-dire l'image d'une sorte d'idéal de pureté, un chant sans chair, inventé à la fin du XIX ${ }^{\mathrm{e}}$ dans un mouvement de restauration catholique (cf. Cheyronnaud 1984) ; au contraire, ici le chant est très corporel, timbré, charnu. Mais un autre élément s'est produit, plus décisif encore tant il est nouveau - même s'il ne se remarque peut-être même pas à l'écoute tellement pour nous, occidentaux, il est naturel : il y a plusieurs voix. Fin du $\mathrm{XI}^{\mathrm{e}}$ siècle, $\mathrm{XII}^{\mathrm{e}}$ siècle, c'est l'invention de la polyphonie - invention tardive en Occident, donc, mais à l'expansion très rapide ensuite. S'il y a une invention qui fait rupture, si on la regarde de façon rétroactive, depuis notre bout de la lorgnette, c'est ce moment décisif, parce qu'il ouvre à l'harmonie. Dès qu'il y a deux voix humaines pensées ensemble, et non pas des chants qui se répondent ou seulement des instruments qui ne font que soutenir une voix, il y a un autre rapport aux intervalles.

\section{Écoute 6}

https://archive.org/details/02perotinorganum4vx

Pérotin-le-Grand : "Viderunt Omnes ", XIII" siècle, Organum à 4 voix ; The Early Music Consort of London dirigé par David Munrow.

Par rapport aux chants tribaux ou religieux essayant d'exprimer l'identité, la puissance, la ferveur ou les aspirations d'un collectif qui se constitue par ces rites mêmes, la polyphonie est l'événement qui change la donne, et en tout premier lieu les rapports entre musique et parole, ouvrant à quelque chose qui commence à ressembler à ce que nous appellerons musique. Cela, à condition d'entendre polyphonie au sens strict où Arom la définissait chez 
les Pygmées Aka, par opposition à la simple superposition de mélodies : des voix construites ensemble, les unes par rapport aux autres. Ce ne sont pas les instruments qui ont introduit la polyphonie, ils sont avant tout percussifs, là pour donner son rythme à la mélopée, même quand ils sont mélodiques. Mais dès que des voix humaines chantent en même temps des mélodies différentes, on entend des intervalles, et très vite en Occident, on va les travailler. On va les travailler parce qu'on les a sous les yeux, fixes : c'est au même moment qu'on les écrit de façon de plus en plus précise et systématique. Comme souvent, les effets de ce changement radical ne sont pas progressifs mais soudains : le premier geste est le bon, à peine l'invention est-elle acquise que tout semble là, en place, dès les premiers produits de la nouvelle technique.

Notons d'abord que ce hoquet, comme on appelle cette forme, est vocalisé sur une seule syllabe : le texte sacré est là, mais décomposé par voyelle, donc incompréhensible (comme plus tard dans les motets du XIV où toutes les voix chantent des paroles différentes) ; la parole devient un code, on voit combien le rapport entre parole et musique est bouleversé. C'est vrai pour le traitement des mots, donc, et pour le rythme, entièrement prédéterminé, mais plus généralement encore, c'est le cas pour les voix elles-mêmes et l'usage de l'écrit : ces musiques sont entièrement pré-calculées (les musiciens contemporains les adorent pour cela). Ni la chanteuse africaine, ni la nonne de Syrie, ni le chanteur populaire de tous les temps et de tous les pays ne pourrait les chanter "d'oreille», il faut les déchiffrer sur le papier pour savoir quelles notes faire.

Autrement dit, à peine la notation n'est-elle plus seulement un moyen mnémotechnique mais qu'elle indique précisément les hauteurs et les durées, qu'est inventé cet objet tout nouveau, un morceau que personne ne pourrait improviser, qu'il faut lire note à note pour être capable d'en restituer l'écriture des voix, comme on dit depuis lors avec raison. Mais il faut réaliser que c'est le contraire de tout ce qu'on a fait jusque-là. Seule la lente familiarisation avec l'harmonie tonale et ses successions d'accords, en reconstruisant un cadre commun et donc la souplesse qu'il réintroduit, redonnera au musicien la possibilité d'improviser ensuite, il faudra attendre Schœnberg et Boulez pour être de nouveau obligé d'avoir l'œil collé à la partition pour savoir quoi jouer. Mais déjà au XIVe siècle, essayez d'improviser un motet de Guillaume de Machaut, sans parler de Dufay puis de l'Ars subtilior qui va suivre en Italie jusqu'au début du XVe siècle, avant le motet "Spem in alium » à quarante voix de Thomas Tallis au $\mathrm{XVI}^{\mathrm{e}} \ldots$

\section{De la musique notée à la musique écrite...}

Écoute 7

https://archive.org/details/8josquinmilleregretz/7-Machaut-DeToutesFlours.mp3

Sylvain Moore / Orlando Consort : "De toutes flours ", ballade de Guillaume de Machaut, XIVe siècle. 
En une génération, au XIIe siècle, on ne va donc plus noter ce qu'on chante, mais chanter puis jouer - ce qu'on écrit. Sur le cas de la musique qui nous préoccupe, c'est ici que l'idée d'une «inversion du pensable » est le plus manifeste, ainsi que son lien avec l'instauration d'une réalité autre grâce à l'écriture - ce qui est l'objet même du livre de Certeau. Un peu de grandiloquence, l'événement le mérite : la musique est née ! Il faudrait écrire "la musique " est née, avec des guillemets, pour souligner que la chose existait bien auparavant, mais pas en tant que telle... Le fait même de l'écrire la fait exister autrement (Goody 1979), et mieux se nommer. C'est cela sa généalogie, son instauration progressive : il serait aussi réducteur de dire que la musique existait déjà mais qu'elle n'avait pas de nom, comme si la nommer en propre n'était qu'un détail, que de dire qu'elle n'existait pas avant d'être baptisée telle. Dès lors, le mouvement est lancé, les compositeurs se différencient des simples musiciens, ce clivage ne faisant ensuite que s'agrandir, qui séparera aussi musique « savante > et musiques 'populaires ) (peut-être vaudrait-il mieux dire musiques qui n'ont pas besoin de présenter leur carte d'identité pour exister ?). La chose est réciproque, bien sûr : les auditeurs vont peu à peu apprendre à entendre la musique comme telle, pour elle-même, puis à apprécier leur musique et à en discuter avec d'autres. Autrement dit, ils vont devenir des amateurs, tandis que s'organiseront les dispositifs nécessaires à une telle transformation, du format des concerts à la construction des salles ou à l'enseignement (cf. Hennion 2004).

C'est pour cela que je préfere parler d'objet musical, au sens où l'on parle d'un objet d'intérêt : il ne s'agit pas de la production de partitions, mais bien de la réorganisation d'un large ensemble de pratiques et de dispositions hétérogènes autour d'une réalité en train de se constituer et d'acquérir une existence propre. Pour autant, est-ce qu'on quitte simplement un univers fait de parole, de rite, de mélopée, de rythme, de religion, pour entrer dans un monde de la musique-pour-la-musique ? Non, ce n'est pas cela le mouvement, parce que dans l'autre sens, tous ces univers se sculptent, se refaçonnent les uns les autres, et que la formation de cet objet musical aux contours plus strictement définis permet en retour de réinvestir ce dont la musique s'est d'abord détachée, et en particulier de reprendre la question de paroles mises en musique, ce que l'on va voir maintenant. Voici un morceau que je trouve presque miraculeux, pour user d'un mot peu sociologique. Il date du tout début du $\mathrm{XVI}^{\mathrm{e}}$ siècle, donc à peine un siècle après l'Ars subtilior.

Écoute 8

https://archive.org/details/8josquinmilleregretz/8-JosquinMilleRegretz.mp3

Hilliard Ensemble : « Mille regrets de vous abandonner » de Josquin des Prés, vers 1510.

Il s'agit de l'un des premiers tubes de la musique. On en a retrouvé des copies dans toute l'Europe, et des transcriptions pour toutes les formations possibles. Si l'écriture est modale au sens un peu scolaire du terme (l'échelle est modale, en mode de mi, les cadences sont plagales, etc.), en revanche il n'y a aucun des accents expressifs de la musique modale : l'espace installé par les quatre voix égales est harmonieux et harmonique, non pas au sens de notre future tonalité réglée par l'enchaînement $7^{\mathrm{e}}$ de dominante-tonique, mais par ses suites 
d'accords parfaits sur des basses en relation de quinte. Les paroles sont profanes, ce qui refait un pont avec les musiques populaires d'antan, et elles sont aussi claires que la progression du contrepoint, entrelaçant les mélismes qui se répondent. Celui qu’on nommait « le Prince de la musique " livre une polyphonie directe, sereine, aussi loin des procédés de l'Ars subtilior du siècle précédent que de la simplicité monophonique d'un chant médiéval : dès qu'on parle de Josquin, le mot ‘ perfection > vient à la bouche de ses contemporains. Je me plais à penser que c'est justement parce que, par rapport à ses prédécesseurs, le sentiment de calme et d'équilibre de cette polyphonie savante lui fait retrouver l'expression et la simplicité de la chanson d'amour, par des moyens qui en sont très éloignés. Après s'être laissées avaler par leur intégration dans la polyphonie complexe et tendue de l'Ars nova, les paroles reprennent leur propre force tout en profitant du nouveau support musical que la polyphonie lumineuse de la Renaissance leur offre.

\section{Des mots qui chantent, des corps qui dansent}

À côté de la parole psalmodiée, je n'ai guère évoqué jusqu'à présent l'autre origine de la musique : la danse. Si les mots prennent vie, rythme, consistance dans le chant, les corps font de même dans la danse, ils se disciplinent et se libèrent à la fois, individuellement et collectivement. Comme pour le rapport parole-musique, plus on remonte dans le temps, moins la distinction danse-musique est pertinente, et là aussi, suivant un geste analogue, une fois son autonomie mieux établie, la musique réélabore son rapport à la danse : comme dans le cas de la parole, elles se renforcent et se réinventent l'une l'autre. L'Église a eu son mot à dire, dans l'affaire, et cette fois la parole et la danse ne sont pas traitées également. Si, reprenant en charge la force du rituel, l'Église a beaucoup ouvré à faire se discipliner l'une par l'autre la parole et la musique sacrées, elle s'est montrée beaucoup plus méfiante vis-à-vis du corps. Elle a vite relégué la danse hors de sa vue - un geste qui a rapproché celle-ci des musiques populaires. Le développement de la musique instrumentale, très liée à la danse, s'est donc fait dans la rue, sur les places, puis dans les salons. Plus latéral par rapport aux pouvoirs, elle s'est moins éloignée des pratiques populaires et moins rapprochée des institutions, lesquelles ont été constamment obsédées par la musique religieuse puis par l'opéra : la musique instrumentale a d'abord été l'œuvre des musiciens eux-mêmes.

L'accent que j'ai mis sur la parole ne doit pas faire oublier en effet que la musique occidentale est tout aussi saturée d'aspects rythmiques que de prouesses de la voix, et ceux-là lui viennent en droite ligne de cette autre moitié de son échafaudage : la danse, c'est-à-dire l'élan du corps, le battement des mains, la puissance de la répétition et de l'alternance de temps inégaux. Le jeu instrumental s'invente ainsi à partir de l'élaboration contrapunctique et harmonique de cette formidable pulsation des corps. Sur cet aspect instrumental aussi, comme dans le cas du rapport entre parole et musique, après un long travail pour maittriser, égaliser, neutraliser le rythme musical, la musique classique a pu revenir vers la danse, 
d'un côté lui apporter la force de ses constructions, et de l'autre lui emprunter en retour les étranges pouvoirs des corps en mouvement. Un seul exemple, mais il est frappant.

\section{Écoute 9}

https://archive.org/details/8josquinmilleregretz/9-SolerFandango.mp3

Scott Ross : « Fandango en ré mineur » de Padre Soler.

Le Padre, toujours endiablé, est cette fois déchaîné. Le mouvement perpétuel que cet extrait interrompt ne cesse de s'intensifier pendant vingt minutes. Mais qui danserait dessus ? La sonate, comprise au sens large sur la durée, des premières courtes pièces italiennes aux monuments romantiques en passant par les suites de danses, c'est une prise de possession progressive des mouvements de la danse par les instruments. Au côté de la maitrise du chant vocal, c'est l'autre parent de la musique classique occidentale. Je ne peux développer ici cette moitié de l'histoire, pourtant indispensable. Heureusement, elle a mieux été étudiée, de Moritz ou Hanslick à Meyer (1956) ou Dahlhaus (1978), surtout sous l'angle de l'invention d'une musique absolue, n'ayant d'autre finalité qu'elle-même ou, pour les sémiologues, d'autre signifié qu'elle-même.

Mon propos se situe en quelque sorte en amont de cela, il se veut moins philosophique qu'anthropologique : selon moi, la question n'est pas la défense et illustration d'une révolution esthétique, mais celle d'une transformation du sensible. Il s'agit moins de la quête de l'art-pour-l'art ou de la musique pure que de la naissance de la musique, tout court, avec l'apprentissage de l'écoute que cela comprend. À l'instar du Padre Soler sur un rythme espagnol, tous les musiciens des XVII et XVIII e siècles s'emparent, explicitement ou non, de danses italiennes, françaises, anglaises pour en faire... de la musique, tout simplement. Mais de la musique instrumentale : nos oreilles ne comprennent plus que les contemporains ne comprennent pas qu'on puisse jouer de la musique sans paroles, qui ne soit pas simplement l'accompagnement rythmique d'une danse. "Sonate, que me veux-tu ? ", il faut prendre au pied de la lettre l'apostrophe de Fontenelle, que nous confondons avec l'affirmation d'une préférence pour l'opéra. Si la formule est apocryphe, rappelle Violaine Anger (2006) dans le livre éponyme qui reprend toute cette histoire, elle exprime bien le fait qu'encore à l'époque, on trouve aberrante l'idée même que des sons tout seuls fassent sens, alors que nous trouvons cela tout naturel. Comme nous l'avons vu dans le cas du chant par rapport à la parole scandée, nous sommes au cœur de ces changements dont l'avènement même met radicalement à distance notre oreille de la leur : le lent travail d'instauration de la musique est inaudible une fois qu'il a été accompli.

\section{L'opéra, l'art politique par excellence?}

Je vais passer plus vite sur la période moderne. En effet, au fur et à mesure qu'elle s'instaure l'histoire de la musique instaurée et celle de son instauration se confondent - c'est là une 
autre façon de dire l'objet de cet article : retrouver les traces qui permettent de dégager l'une de l'autre la production "produite " et la production " produisante ", comme les appelait Certeau. Comment faire la différence entre l'« histoire de la musique » des manuels pédagogiques, présentant un défilé de compositeurs, de genres et d'œuvres comme s'il allait de soi qu'ils appartiennent tous à un même monde, celui de la musique; et une histoire qui ne tienne pas la musique pour acquise, ni aucune de ses particules élémentaires, mais suive pas à pas la façon dont elle s'est faite musique ${ }^{7}$, dans la pluralité même de ses formes, tout en effaçant derrière elle les traces de sa propre musicalisation ?

Pour le montrer, je vais m’appuyer sur deux exemples caractéristiques, l'opéra et la mélodie française : avant de conclure, cela remettra la focale sur le rapport parole-musique. Revenons d'abord sur un moment très important et complexe par rapport à cette question, la naissance de l'opéra. La musique s'est développée, elle est dans l'église mais aussi dans la rue, des formes populaires ont été reprises et sophistiquées, on dispose d'un majestueux répertoire de formes religieuses, de grands motets, de messes, de chorals allemands... et le théâtre ? Des paroles sur scène, rythmées, scandées, accentuées, expressives, pourquoi y aurait-il ce spectacle d'un côté, et de l'autre une musique qui ne servirait qu'à prier ou à faire danser les paysans ? Il faut réunir les deux ! Les Italiens relèvent le défi, l'histoire des deux Camerate à la fin du XVIe siècle est connue, et là encore ce mouvement est hautement réflexif, très conscient de ce qu'il invente. Si désormais, depuis nos anachronismes actuels, l'opéra n'est qu'un genre parmi les autres, il faut réaliser que du XVII e siècle à la toute fin du XIX'e les choses ont été bien différentes : la musique, c'est l'opéra. J'exagère un peu, il y a le piano, les symphonies, mais dès que la musique a acquis ses propres institutions, ce qui compte vraiment, pour le public aussi bien que pour le pouvoir, et aussi pour la plupart des compositeurs, c'est l'opéra.

Ne s'agit-il pas là encore d'un autre retour de la parole dans la musique ? En quoi le triomphe de ce genre mêlé, plein de facilités, tant théâtrales et romanesques que vocales, avec ses airs brillants et ses vedettes, s'inscrit-il dans l'histoire dans sa version officielle, celle d'une autonomisation progressive de la musique-pour-la-musique et du lent triomphe de la musique pure ? J'ai pris ce cas pour cela : non pas tant pour souligner ce mouvement d'autonomisation de la musique, d'autres l'ont fait (pour ma part, je l'interprèterais plus volontiers comme l'instauration de la musique en tant que musique), que pour insister sur le processus réciproque, moins souvent relevé, à savoir la capacité de la musique, ainsi de mieux en mieux identifiée, à s'infiltrer en retour dans les autres réalités, et non pas à se fermer dans son univers propre. Qu'y a-t-il donc, dans un opéra ? Un jeune premier amoureux d'une princesse, ou au contraire d'une paysanne, qu'un vieux roi vient contrarier, tandis que les masses populaires se révoltent et que se trament des complots dans les allées du pouvoir... On pourrait remonter au rôle de l'opéra à la cour de Versailles, mais même dans la France du XIX siècle, Jane Fulcher (1988) a très bien montré combien ce qu'on appelle aujourd'hui un genre bourgeois l'est en effet : bourgeois non pas au sens anachronique où il serait facile et sentimental, mais au sens où il contribue activement à forger l'identité de cette classe alors conquérante. Il est à peine exagéré de dire que la bourgeoisie s'invente avec l'opéra. Légitimité du pouvoir, place de l'individu par rapport aux masses, de l'ordre par 
rapport à la justice et à la liberté, relation entre l'État et la religion, naissance du sentiment national, il n'y a qu'à passer en revue les titres des grands succès de l'opéra français d'alors, dans toute l'Europe, pour voir combien l'image d'un genre conformiste est une projection moderniste du XX $\mathrm{XX}^{\mathrm{e}}$ siècle sur le XIX ${ }^{\mathrm{e}}$ : mièvres, La Juive (à l'acte 3, on évite de justesse un pogrom), Les Huguenots (sur le massacre de la Saint-Barthélémy), La muette de Portici (un rôle-titre muet, dans un opéra...), dont la Belgique reprendra un chœur lorsqu'elle se soulève... D’Orfeo à Tristan, de Don Juan à Pelléas, l'opéra met en scène la difficile affirmation du sujet individuel face au poids des contraintes sociales, et à l'âge des révolutions, ces conflits sont directement politiques. Verdi ne fait rien d'autre, dont le nom devient le symbole de l'unification de l'Italie. On dit que Napoléon III envoyait des espions à l'Opéra guetter les réactions de ce dont se moquera si bien Offenbach dans La belle Hélène, l'، opinion publique , qui naît à cette époque.

Or, cette force expressive, à quoi d'autre tient-elle qu'au mélange étroit, et sous une forme inédite, des éléments que l'opéra refond dans un moule qui les transforme tous : parole sur-personnalisée par le chant, chœurs donnant une sourde présence à la puissance et à la violence potentielle du peuple, mise en scène théâtrale jouant sur la séduction des corps et les mouvements collectifs, écritures orchestrales et récitatifs très particuliers au genre ?

\section{Prima la parola, prima la musica?}

Si l'on veut resserrer la focale pour viser le lien entre parole et musique, vers la réalisation musicale concrète de la mise en théâtre d'un rôle chanté, pour montrer qu'à chaque niveau s'ouvrent des options différentes, l'exemple de la longue dispute entre l'opéra français et l'opéra italien s'impose : elle ne porte que là-dessus, quitte à ce que les rôles s'inversent entre le début et la fin. Du côté italien, prima la parola, comme le réclame la Camerata au début de l'histoire, ou prima la musica, comme le revendiquera Gianni Schicchi ? Dès ses débuts, l'opéra français, lui, pourtant inventé par Lully, un Italien, est avant tout du théâtre. Il se bat pour que la musique soit un moyen de mettre en valeur des paroles, comme un poème les fait entendre avec plus de force expressive et d'intensité émotionnelle en leur donnant de l’ordre, en les faisant sonner, en insistant sur leur matérialité.

Écoute 10

https://archive.org/details/8josquinmilleregretz/10-CharpentierCidLedroit.mp3

Ledroit, Henri / Ricercar Consort : «Les stances du Cid » de Marc-Antoine Charpentier.

Musique sublime, assurément, entièrement subordonnée au texte dont elle surligne avec un art consommé les moindres reliefs. Les Italiens qui viennent à Paris se plaignent en sortant de la salle : cela fait trois heures qu'ils entendent des récitatifs, et toujours pas le début d'un air... Alors que s'invente en France ce que je présentais sur un mode encore abstrait plus haut, l'art de donner plus de consistance à un texte avec des moyens musicaux entièrement 
tournés vers l'expression (accents, rythmes inégaux, ruptures de tempo et de puissance, respirations, etc.), de l'autre côté des Alpes les Italiens ne se contentent pas d'aimer le chant sans accorder aucune importance au texte, comme les Français les en accuseront : ils le réinventent, ce chant. Dans la direction opposée de celle qu’ont empruntée les Français (il faut se rappeler que chacun va sans cesse visiter, inviter, admirer et jalouser son voisin latin), ce que les Italiens découvrent, puis exploitent et travaillent, c'est tout autre chose qu'un chant au service d'un poème, c'est la voix elle-même. Ils font véritablement de la voix un instrument, et non le moyen d'une parole. Alors que les ténors italiens chantaient encore leurs aigus en voix de tête dans Verdi au XIX ${ }^{\mathrm{e}}$ siècle (comme devraient le faire aujourd'hui les ténors dans l'opéra français), le travail sur l'ouverture du gosier, l'abaissement du larynx, l'appui sur le souffle, l'élargissement de la voix de poitrine les ont fait passer en un siècle de Bellini à Puccini : de l'émotion aussi, assurément, mais à partir d'une distribution entre musique, texte et voix terme à terme opposée.

Pour autant, aucune n'est plus « naturelle ` que l'autre : dans les deux cas, on a affaire à la lente fabrication d'un genre, allant de ses artistes à son public. Ni l'extrait précédent ni celuici ne ressemble à de la mélodie ou du lied, on a chaque fois clairement affaire à du théâtre chanté. Mais par rapport aux stances du Cid, l'auditeur le plus sourd entendrait l'opposition entre ces deux définitions du genre opéra ${ }^{8} \ldots$

Écoute 11

https://archive.org/details/8josquinmilleregretz/11-PucciniToscaELucevanLeStelleKaufmann.mp3

Jonas Kaufmann / Prague Philharmonic Orchestra : "E lucevan le stelle » de Tosca de Giacomo Puccini.

\section{Chanter une chanson, mettre en musique un poème...}

Moins pour conclure que pour ouvrir vers d'autres interrogations, je vais traiter d'un dernier contraste intéressant, qui rapproche du thème de la chanson. Il oppose cette fois deux façons de mettre de la musique le long d'un texte français, que j'enchaîne sans transition.

Écoute 12

https://archive.org/details/8josquinmilleregretz/12-Ferr\%C3\%A9AvecLeTemps.mp3

Léo Ferré : "Avec le temps », 1970.

https://archive.org/details/8josquinmilleregretz/13-Faure\%CC\%81SouzayGreen.mp3

Gérard Souzay (baryton) / Dalton Baldwin (piano) : "Green », Op. 58-3 de Gabriel Fauré, extrait des Cinq mélodies de Venise.

Dans le fil des instaurations contrastées d'assemblages entre musique et paroles que j'ai décortiquées auparavant, comment analyser le premier extrait, sans doute la mélodie la plus 
déprimante du chanteur? Le plus intéressant est sans doute l'incapacité où elle nous place de le faire. Ce n'est pas un reproche. Je me souviens qu'en musicologie à la Sorbonne, dans les années 1970, un professeur voulant bien faire nous demandait d'analyser "Satisfaction ", des Rolling Stones, en mobilisant les variables musicologiques qu'il était chargé de nous apprendre. Nous donnions les accords, le rythme, l'organisation couplets-refrain, mais il était évident pour nous tous que rien de ce qui faisait la force de l'un des plus grands tubes du rock ne ressortait de cela. On ne peut analyser une musique quà partir des éléments qu'elle offre elle-même, éléments difficiles à appréhender parce que, précisément, chacune les recompose à sa façon, et que cette recomposition est sans doute l'un des ressorts principaux de sa reconnaissance.

Ce « tube > de Ferré nous met dans la même situation que des étudiants en musicologie laissés perplexes par leur outillage analytique. Il y a bien la diction d'un diseur de poèmes extraordinaire, à la voix un peu cassée, mais ni les paroles si on les dit sans la musique, ni les accords banals qui sont de ceux que tous les arrangeurs utilisent, ni même la mélodie ne sont bien marquants, isolément. Et pourtant... comment rendre compte de la force émotive qui en a fait l'une des chansons les plus marquantes de sa génération ? Peut-être peut-on y déceler l'inverse total de ce que j'ai montré, une façon d'éviter tout recours à des éléments musicaux identifiés, séparés, réélaborés (d'où la vanité de l'analyse), au profit d'une personnalisation maximale de l'interprète-auteur, d'un effort pour faire se confondre en une performance radicalement synthétique sa propre chanson avec son corps, sa vie, sa voix. Mais comment prouver cela, sinon constater qu'en effet dans l'histoire de la chanson, dire Ferré évoque aussitôt "Avec le temps ». Dans ces conditions, ‘ analyser > une musique ne consisterait pas à projeter sur elle des catégories toutes faites (paroles, musique, mais aussi accords, tonalité, rythmes, formes, etc.), comme si elles allaient de soi, rendues trop évidentes par leur usage même. Cela supposerait au contraire de se redemander chaque fois comment elle a réussi à mettre en œuvre, comme on dit joliment, une définition propre de ce qui ‘ fait musique `.

Avec une mélodie de Fauré ou de Debussy à la fin du XIX', on est à l'opposé de ce que je viens de décrire trop grossièrement à propos de Ferré, alors même qu'il s'agit dans les deux cas de textes français mis en musique pour être chantés. Il y a des moments de l'histoire où un style semble se concentrer sur lui-même : dans la proximité apparente de ses œuvres, mais dans le contraste de leur moindre détail lorsqu'elles sont prises une à une, ce style ou ce genre prend une densité exceptionnelle, souvent sur une période assez courte, comme s'il devait s'épuiser. Ce n'est d'ailleurs nullement l'apanage des genres classiques : c'était le cas du rock des années 1960 dont je parlais, du rap des années 1990 ou du bebop après 1945, comme de l'éruption romantique du milieu du XIX siècle. Plus près de ce qui nous concerne ici, cela a aussi été le cas du lied germanique, dont la mélodie française s’inspire et se décale en même temps. En l'occurrence, il s'agit bien d'une musique savante, extrêmement savante même, comme dans le cas du madrigal italien au XVe siècle, des airs de luth français et anglais au XVI ${ }^{\text {e }}$, ou des chorals et du contrepoint allemand au XVII ${ }^{\text {e }}$, bref de toutes ces musiques que ceux qui les connaissent mal trouvent ‘ toutes pareilles `. C'est là qu'on 
trouve des merveilles. Sans être beaucoup plus capable d'expliquer pourquoi une musique est belle, cette fois l'analyse peut se déployer sans retenue : à un moment où la poésie ellemême était dans un état analogue, les mélodistes, tout en se guettant les uns les autres, ont produit par centaines des mélodies dont les moindres détails ont été sculptés, juste au point d'intersection qui nous intéresse ici : la jonction entre le son d'un mot, son sens, sa place dans une phrase, et la ligne mélodique et harmonique dans lequel il s'insère. La même chose vaut pour les interprètes, obligés de réinventer leur voix pour chaque mélodie, sans pouvoir recourir à leur arsenal habituel : si c'est trop vocal, cela fait opéra, si c'est trop précieux, cela fait salonnard, si l'on chante sans penser à chaque mot, à chaque syllabe du texte, à chaque consonne, on s'ennuie... Non, il faut trouver le ton juste, pour cette mélodie. Les impossibles voyelles françaises, on, un, an, $u, \hat{e}, e ́, e u h$, l'arrêt imposé par les consonnes, les liaisons, la longueur d'un mot ou la sécheresse d'une finale, tout a servi de ressources aux compositeurs pour articuler avec une précision d'orfevre le son et le sens, et le bon interprète retrouve un par un, tout en les réinterprétant, ces petits diamants qui font toute la différence Gérard Souzay n'a pas chanté de la mélodie française, c’est elle qui lui a inventé sa voix!

\section{Conclusion}

Cet article expérimental a tenté, sur un mode plus tâtonnant qu'affirmatif, d'aborder l'une par l'autre deux questions, qui ne se situent pas au même niveau.

D'une part, comment faire autrement de l'histoire de la musique, en évitant le double piège du positivisme, qui prend les choses du passé pour des faits que les méthodes scientifiques sauront de mieux en mieux cerner, et du présentisme, qui ne fait des choses du passé que la projection de nos conceptions, comme si elles pouvaient tout supporter. J'ai essayé d'esquisser cette autre histoire de la musique, tout au présent, mais pleine de ses pluralités possibles.

D'autre part, comment exploiter le travail même des interprètes au fil du temps, qui sans cesse rejouent des musiques venues d'autres temps et d'autres lieux, pour apprendre d'eux sur un mode sensible une double expérience paradoxale, quelque chose comme une distance qui instaure un accès, au lieu de le fermer ("nous ne retrouverons pas le passé ») ou de l'annuler ("nous l'écoutons tel qu'il était»). Pour le dire autrement, non pas se réapproprier aujourd'hui toutes les musiques comme dans un rayon de supermarché, mais s'appuyer sur la perte irrémédiable de leurs effets passés, et de l'écoute existant en leur temps, pour prendre conscience d'écoutes différentes, et apprendre à en jouir autrement, aujourd'hui.

Au moyen d'écoutes réorientées, l'effort a consisté à sortir des « Histoires de la musique , qui malgré leur nom font tout sauf de l'histoire, alignant formes, compositeurs et dates le long d'une échelle de temps fixe, comme si l'on pouvait ainsi décrire les avatars d'un objet universel qui ne ferait qu'évoluer en présentant des aspects variés au fil du temps. Car réciproquement, parler, chanter, musiquer, écouter, rien de cela ne saurait avoir un sens fixe, indépendant des performances qui, morceau par morceau, forme par forme, genre par genre, ont réussi à forger entre ces actes une cohérence plus ou moins stable et durable. Revenir sur 
l'engendrement réciproque de ces éléments musicaux imposait donc d'essayer d'oublier ce qu'ils sont devenus, pour insister sur les ruptures plus que sur une continuité finaliste ayant inexorablement débouché sur notre modernité.

À ce titre, le cas des rapports entre musique et parole est particulièrement instructif : dans la mesure où il n'y a pas la musique et la parole, d'abord, puis leurs rapports et leur évolution, il nous a fallu suivre de loin ce travail de formation et de transformation mutuelles, et nous réapproprier ses résultats sur un autre mode, alors même que, à mesure qu'il aboutit à une forme qui s'instaure, ce processus a fait disparaitre les états antérieurs des éléments qu'il a redéfinis. À côté de multiples autres éléments (matériels, corporels, rituels, sociaux, institutionnels...), des notions plus typiquement musicales comme la scansion, le rythme, la mélopée, les accents ont forgé des mondes pluriels, souvent étanches les uns aux autres (le fait même d'écouter plusieurs musiques est un trait de notre époque), et ces mondes se sont peu à peu eux-mêmes identifiés comme étant musicaux. Comme au second degré, réécouter ces musiques du passé ne consiste pas à se plonger dans le passé, mais au contraire à les entendre comme appartenant au passé. Donc à prendre conscience par les sens d'une distance radicale à une musique qui ne sera jamais la nôtre : le paradoxe, c'est que ce geste même peut nous ouvrir la possibilité d'en avoir néanmoins une autre écoute, actuelle, la nôtre cette fois. L'écoute du passé a elle-même une histoire.

\section{Notes}

1 Antoine Hennion est professeur au Centre de sociologie de l'innovation (CSI) Mines-ParisTech (PSL Université / CNRS, Paris).

2 Rien de plus juste que le double sens du mot " réaliser ", à la fois comprendre et faire les réalités.

3 Même si l'on peut lire le livre d'Austin, qui devait être suivi d'un second qu'il n'a jamais écrit, comme un long cheminement rendant compte pas à pas de la découverte des conditions de félicité de ces énoncés performatifs : liste toujours plus ouverte, qui le fait abandonner la clarté de son dualisme initial au profit d'une théorie beaucoup plus graduée des actes de langage. Cf. aussi Fraenkel 1992 et la reprise récente de ce vieux problème par Barbara Cassin 2018.

4 Les pragmata sont les " choses dans leur pluralité », en tant qu'elles ne sont pas données (James 1909, 210).

5 C'est aussi au sens de le faire passer, ce passé qui ne passe pas, pour la part violente, dominatrice, traumatique ou honteuse qu'il a toujours aussi, tant qu'il n'est pas formulé.

6 Signalons au passage que tous ces extraits viennent de disques, bien sûr : on voit la roublardise qu'implique un retour en des temps anciens que seuls les moyens modernes rendent possible. L’‘ authenticité > requiert plus de médiations, non pas moins (cf. Hennion 2007).

7 ...ou dont elle s'est refaite musique, sur un autre mode, hautement réflexif : qu'on pense au renouveau de la musique dite baroque (Hennion 2007, 219-267).

8 Il est vrai que je triche un peu en sautant directement du XVII siècle à la fin du XIX (et en faisant chanter Puccini par un ténor allemand : il n’y a plus de chant national !). 


\section{Bibliographie}

Anger, Violaine : "Sonate, que me veux-tu ?". Pour une histoire du signe. Paris : ENS Éditions, 2006.

Arom, Simha : Polyphonies et polyrythmies instrumentales d'Afrique centrale. Structure et méthodologie. 2 vol. Paris : SELAF, 1985.

Austin, John Langshaw : Quand dire c'est faire. Paris : Le Seuil, 1970 [1962].

Boquet, Damien / Dufal, Blaise / Labey, Pauline (éds) : Une histoire au présent. Les historiens et Michel Foucault. Paris : CNRS éditions, 2013.

Cassin, Barbara : Quand dire c'est vraiment faire. Homère, Gorgias et le peuple arc-en-ciel. Paris : Fayard, 2018.

Certeau, Michel de : L'absent de l'histoire. Tours : Mame, 1973.

Certeau, Michel de : L'écriture de l'histoire. Paris : Gallimard, 1975.

Cheyronnaud, Jacques : Le lutrin d'Église et ses chantres au village (XIXe-XXe siècles). Approche d'un service public musical. Thèse en ethnologie. EHESS Paris 1984.

Cheyronnaud, Jacques : "Ethnologie et musique : l'objet en question ». In : Ethnologie française 27,3 (1997), 382-393, http://www.jstor.org.inshs.bib.cnrs.fr/stable/40989904 (consultation 10.12.2019).

Dahlhaus, Carl : Lidée de la musique absolue. Une esthétique de la musique romantique. Genève : Contrechamps, 1997 [1978].

Despringre, André-Marie (éd.) : Le chant traditionnel, question de sens et de style : interdisciplinarité ethnomusicologique et ethnolinguistique. Paris : L'Harmattan, 2019.

Fauquet, Joël-Marie / Hennion, Antoine : La grandeur de Bach. L'amour de la musique en France au XIX siècle. Paris : Fayard, 2000.

Foucault, Michel : L'archéologie du savoir. Paris : Gallimard, 1969.

Foucault, Michel : Dits et écrits, 1954-1988. 4 vol. Éds Daniel Defert / François Ewald. Paris : Gallimard, 1994.

Fraenkel, Béatrice : La signature. Naissance d'un signe. Paris : Gallimard, 1992.

Fulcher, Jane : Le grand opéra en France : un art politique, 1820-1870. Paris : Belin, 1988.

Goody, Jack : La raison graphique. Paris : Minuit, 1979.

Hanslick, Eduard : Du beau dans la musique. Paris : Bourgois, 1986 [1854].

Hartog, François : Régimes d'historicité. Présentisme et expérience du temps. Paris : Le Seuil, 2003.

Hennion, Antoine : «Une sociologie des attachements. D'une sociologie de la culture à une pragmatique de l'amateur ". In : Sociétés 85,3 (2004), 9-24.

Hennion, Antoine. La passion musicale. Une sociologie de la médiation. Paris : Métailié, 2007 [1993]. Hennion, Antoine : «Soli Deo Gloria. Bach était-il un compositeur? ». In : Gradhiva 12 (2010), 4055, http://gradhiva.revues.org/1837 (consultation 10.12.2019).

Hennion, Antoine : "Jouer, interpréter, écouter. Pratiquer la musique, ou la faire agir ? ". In : Fourmentraux, Jean-Paul (éd.) : L’ère post-média. Humanités digitales et cultures numériques. Paris : Hermann, 2012, 87-102. 
Hennion, Antoine : "Enquêter sur nos attachements. Comment hériter de William James ? ". In : SociologieS (2015), Dossier Pragmatisme et sciences sociales : explorations, enquêtes, expérimentations, http://sociologies.revues.org/4953 (consultation 10.12.2019).

Hennion, Antoine / Monnin, Alexandre : "Sous la dictée de l'ange. Enquêter sous le signe d'Étienne Souriau ». In : Courtois-l'Heureux, Fleur / Wiame, Aline (éds) : Étienne Souriau. Une ontologie de l'instauration. Paris : Vrin, 2015, 131-156.

James, William : The Meaning of Truth. NY : Longmans Green \& Co, 1909.

Lenclud, Gérard : «La tradition n’est plus ce qu'elle était... ». In : Terrain 9 (1987), 110-123, http:// terrain.revues.org/3195 (consultation 10.12.2019).

Meyer, Leonard B. : Emotion and Meaning in Music. Chicago: University of Chicago Press, 1956.

Moritz, Karl Philipp : Le concept d'achevé en soi et autres écrits, 1785-1793. Paris : Presses universitaires de France, 1995 [1785-93].

Rouget, Gilbert : La musique et la transe. Paris : Gallimard, 1985.

Souriau, Étienne : Les différents modes d'existence. Paris : PUF, 1956.

Veyne, Paul : Comment on écrit l'histoire. Paris : Le Seuil, 1971.

\section{Discographie}

Banda Gbambiya : "Nginza ngiriki ". In : République centrafricaine. Musiques de xylophones (Collection du CNRS et du Musée de l'homme). Le Chant du monde LDX 274932, 1992 (CD). Ensemble Organum / Marcel Pérès : "Sanctus ». In : Ensemble Organum / Marcel Pérès : Chant corse. Manuscrits franciscains (XVII'-XVIII siècles). Harmonia Mundi HMC 901495, 1994 (CD).

Ferré, Léo : «Avec le temps ». In : Ferré, Léo : Avec le temps. Barclay 841017-2, 1998 (CD).

Hilliard Ensemble : «Mille regrets de vous abandonner». In : Des Prés, Josquin : Motets et Chansons. Virgin Veritas VER 5613022, 1996 (CD).

Kalthoum, Oum : "Afdihi in hafidza el hawa ». In : Tout Oum Kalthoum. Soldore SOL 533, 1984 (CD).

Kaufmann, Jonas / Prague Philharmonic Orchestra : "E lucevan le stelle »(Giacomo Puccini). In : Kaufmann, Jonas / Prague Philharmonic Orchestra : Romantic Arias. Decca Music 475 9966, 2008 (CD).

Ledroit, Henri / Ricercar Consort : "Les stances du Cid ». In : Charpentier, Marc-Antoine : Orfée descendant aux Enfers. Ricercar RIC 037011, 1987 (CD).

Moore, Sylvain / Orlando Consort : "De toutes flours". In : Machaut, Guillaume de : Dreams in the Pleasure Gardens. Machaut Chansons. Deutsche Grammophon Archiv 457618-2, 1998 (CD).

Munrow, David / The Early Music Consort of London : «Viderunt Omnes ( (Pérotin-le-Grand). In: Munrow, David / The Early Music Consort of London : Music of the Gothic Era. Archiv Produktion 415 292-2, 1985 (CD).

Puccino, Oxmo : "Visions de vie ». In : Puccino, Oxmo : Opéra Puccino. Delabel 8457861, 1998 (double 33 tours). 
Ross, Scott : "Fandango en ré mineur ». In : Padre Soler : Fandango / 9 Sonates. Erato-Disques 2292 45435 2, 1990 (CD).

Sœur Marie Keyrouz / Marcel Pérès : "Exapostilation de l'Office de Mardi Saint ». In : Les Très Riches Heures du Moyen Âge (A Medieval Journey). CD 1. Harmonia Mundi HMX 290649, 1995 (6 CD).

Souzay, Gérard / Dalton Baldwin : "Green ». In : Fauré, Gabriel : La Bonne Chanson. Philips 420775-2, 1984 (CD). 